\title{
Comprehensive analysis of multi Ewing sarcoma microarray datasets identifies several prognosis biomarkers
}

\author{
XUQING YIN ${ }^{1 *}$, JIUBO SUN $^{2 *}$, HAIYANG ZHANG ${ }^{3}$ and SHUAI WANG ${ }^{4}$ \\ Departments of ${ }^{1}$ Traumatic Orthopaedics, ${ }^{2}$ Radiation Oncology and ${ }^{3}$ Microinvasive Othopaedics, Central Hospital of Zibo, \\ Zibo, Shandong 255036; ${ }^{4}$ Department of Spine Surgery, Jining No. 1 People's Hospital, Jining, Shandong 272000, P.R. China
}

Received January 16, 2018; Accepted July 25, 2018

DOI: $10.3892 / \mathrm{mmr} .2018 .9432$

\begin{abstract}
Ewing sarcoma (ES) is a common primary malignancy in children and adolescents. Progression of treatment methods hasn't contributed a lot to the imrovement of prognosis. To identify potential prognostic biomarkers, a meta-analysis pipeline of multi-gene expression datasets for ES from the Gene Expression Omnibus (GEO) was performed. Three datasets were screened and differential expression genes (DEGs) in ES samples compared with normal tissues were identified through limma package and subjected to network analysis. As a result, 1,470 DEGs were obtained which were mainly involved in biological processes associated with immune response and transcription regulation. Network analysis obtained 22 core genes with high network degree and fold change. Kaplan-Meier analysis based on ES datasets from The Cancer Genome Atlas identified five genes, including glycogen phosphorylase, muscle-associated, myocyte-specific enhancer factor $2 \mathrm{C}$, tripartite motif containing 63, budding uninhibited by benzimidazoses 1 and Ras GTPase-activating protein 1, whose altered expression profiles are significantly associated with survival. Changes of their expression values were further confirmed through RT-qPCR in ES cell and normal cell lines. Those genes may be considered as potential prognostic biomarkers of ES and should be helpful for its early diagnosis and treatment.
\end{abstract}

\section{Introduction}

Ewing sarcoma (ES) is an aggressive sarcoma of bone and/or soft tissue with a peak incidence in children and young adults, it is the third most common malignant primary bone

Correspondence to: Dr Shuai Wang, Department of Spine Surgery, Jining No. 1 People's Hospital, 99 Shixian Road, Jining, Shandong 272000, P.R. China

E-mail: shuaiwjn@outlook.com

*Contributed equally

Key words: Ewing sarcoma, gene expression omnibus, network, prognosis, the cancer genome atlas tumor, following osteosarcoma and chondrosarcoma $(1,2)$. Over the past decades, efforts have been made to maximize the chance of cure and pathogenesis of ES through collaboration among clinicians, pathologists, and biologists (3). The overall survival (OS) for ES patients with localized disease is $\sim 70 \%$, but the OS of patients with metastatic disease is only $\sim 30 \%$ (4). Further efforts should be made to improve these outcomes, especially for patients with metastatic and recurrent ES.

Efforts of researchers and clinicians have advanced the understanding of ES oncogenesis and the genetic predisposition for developing ES (5). ES is defined by a balanced translocation that involves the Ewing sarcoma breakpoint region 1 (EWSR1) gene located on chromosome 22, and a member of the E26 transformation-specific (ETS) family of transcription factors that mainly contain the friend leukemia integration 1 (FLII) and EST-related gene (ERG) genes (6). Two types of translocation are generally observed on the molecular analysis of ES samples. The first most common type, accounting for $85 \%$ of the translocations, occurs when the EWSR 1 fuse to the FLII gene which is located on chromosome 11, resulting in an EWSRI-FLII fusion gene (7). The second most common translocation in ES, occurs when the EWS gene fuses to another member of the ETS transcription factor family, $E R G$, located on chromosome 21, resulting in an EWSR 1-ERG fusion gene $(8,9)$. Besides FLII and ERG, other members of the ETS transcription factor family that can act as partners for $E W S R I$ are ETV, ETV4, and FEV (10-12). These fusion oncoproteins might serve as potential diagnostic markers and therapeutic targets for ES. However, several reports demonstrated that in addition to the expression of FLII in ES, it can also be detected in other neoplasms including lymphoblastic lymphomas, Merkel cell carcinoma, desmoplastic small round cell tumor, and synovial sarcoma, endothelial cells and lymphocytes also normally express FLII (13-15). To date, no specific and accurate molecular markers have been established for the early diagnosis and treatment of ES, and therefore identification of new molecular markers is urgently needed.

In the present study, a meta-analysis of several ES transcriptome datasets from the Gene Expression Omnibus (GEO) was performed. Differential expressed genes (DEGs) in ES compared with normal tissues were identified and subjected to network analysis. A Kaplan-Meier analysis of core genes networks was performed and several survival-associated 
genes were identified that could act as potential markers for the prognosis of patients with ES.

\section{Materials and methods}

Datasets. With the keyword of 'Ewing Sarcoma' and restriction of organism='Homo sapiens', platform='GPL570' and attribute $=$ 'Tissue', a total of three datasets with the accession number of GSE34620 (5), GSE17618 (16) and GSE17674 (16) were obtained from the GEO (www.ncbi.nlm.nih.gov/geo/). There are 117 ES tissue samples in GSE34620 and 44 ES tissue samples in GSE17618 and without normal samples aside from the 18 in GSE17674. For GSE17674, a total of 44 ES tissue samples and 18 normal skeletal muscle samples were included. Table I demonstrates detailed information of datasets used in the present study.

Microarray preprocessing. The raw datasets were firstly normalized prior to differential expression analysis. In brief, the CEL files were imported into R (www.r-project.org/), a free access statistics software, to conduct batch normalization with the sva package (17). Probe level expression values were transformed to gene level based on the microarray annotation file. For genes corresponding to multi probes, the mean expression value was used.

Differential expression analysis. The limma package (18) was used for the identification of genes with aberrant expression profiles in ES compared with normal tissues. The t-test and FDR correction were used to test significance of expression differences between ES and normal tissues, and only those genes with adjusted $\mathrm{P}<0.05$ and $\mid \log 2$ Fold Changel $>1$ (fold change $>2$ or $<0.5$ ) were considered with a significantly differential expression.

Functional enrichment analysis. To explore functions involved in differential expression genes (DEGs) in ES samples, the Database for Annotation, Visualization and Integrated Discovery (DAVID, david.ncifcrf.gov/) (19) was used for the functional enrichment analysis, and Gene Ontology (GO) terms and Kyoto Encyclopedia of Genes and Genome (KEGG) pathways with P-value $<0.05$ were screened out. In addition, to interpret associations among those functions, the enrichmentMap plug-in (20) of Cytoscape software (21) was used to perform crosstalk analysis of Biological Process (BP) terms.

Network analysis. By combining the network deposited in Protein Interaction Network Analysis (PINA, cbg.garvan. unsw.edu.au/pina/) (22) and Menche's study (23), interaction pairs among DEGs were screened. Additionally, the MCODE plug-in of Cytoscape software was used to conducted modular analysis of the whole network.

Kaplan-Meier analysis. Hub network genes (genes with high degree) should serve an important role in ES progression for the high number genes directly interacting with them. In the present study, a Kaplan-Meier analysis for hub network genes was conducted based on another ES-associated dataset downloaded from The Cancer Genome Atlas (TCGA; cancergenome.nih.gov/) to identify genes significantly associated with ES overall survival (OS).

Reverse transcription-quantitative polymerase chain reaction $(R T-q P C R)$. Total RNA was isolated from ES cell lines A673 and normal mesenchymal stem cells (MSC, from Cyagen Biosciences, Guangzhou, China) using an RNeasy ${ }^{\circledR}$ Mini kit (Qiagen GmbH, Hilden, Germany) according to the manufacturer's protocol. First-strand cDNA was synthesized from $11 \mu \mathrm{g}$ of total RNA using the Transcript or First Strand cDNA Synthesis kit (Roche Diagnostics, GmbH, Mannheim, Germany) according to the manufacturer's protocol. RT-qPCR reactions were performed on an ABI 7500 real-time PCR system (Applied Biosystems; Thermo Fisher Scientific, Inc., Waltham, MA, USA) using the following procedure: $95^{\circ} \mathrm{C}$ for $10 \mathrm{~min}$, followed by 40 cycles of $95^{\circ} \mathrm{C}$ for $30 \mathrm{sec}$ and $60^{\circ} \mathrm{C}$ for $1 \mathrm{~min}$. Primers used for glycogen phosphorylase (PYGM) are $\left(5^{\prime}->3^{\prime}\right)$ : Forward primer, 5-TTTCACACTCGTAAAGGACCG CAAT-3, and reverse primer, 5-TGTTCTGTAGCGTCCGTC CCATATA-3. Primers used for myocyte-specific enhancer factor $(M E F) 2 C$ are $\left(5^{\prime}->3^{\prime}\right)$ : Forward primer, 5-TGGGTTGAT GAAGAAGGCTTATGAG-3, and reverse primer, 5-TAAGGC CCTTCTTTCTCAACGTCTC-3. Primers used for Tripartite Motif Containing (TRIM)63 are $\left(5^{\prime}->3^{\prime}\right)$ : Forward primer, 5-AAGCCAGTGGTCATCTTGCCGT-3, and reverse primer, 5-CGTACACTCCGTGACGATCCATGA-3. Primers used for BUB1B, budding uninhibited by benzimidazoses $(B U B 1 B)$ are $\left(5^{\prime}->3^{\prime}\right)$ : Forward primer, 5-GTATAAACCACATCCTAA GCACCAG-3, and reverse primer, 5-CTCTGCACTGGTCAA TAGCTCGGCT-3. Primers used for Ras GTPase-activating protein $(R A C G A P) 1$ are $\left(5^{\prime}->3^{\prime}\right)$ : Forward primer, 5-TGGCAA ATTATCTCTGAAGTGTCGA-3, and reverse primer, 5-CTC TTTGCTCAATCTCATTTACACA-3. GAPDH was used as an internal control. The $2^{-\Delta \Delta \mathrm{Cq}}$ method was used for data analysis (24).

\section{Results}

DEGs. Through the thresholds of adjusted $\mathrm{P}<0.05$ and Ilog2Fold Changel $>1$, a total of 1,470 DEGs were obtained in ES samples compared to normal samples with 984 upregulated and 486 downregulated genes. Fig. 1A shows the heatmap of the top 100 most significant genes with green and red color representing low and high expression levels, respectively. Fig. 1B shows the distribution of DEGs with the green and red dots indicating down- and upregulated genes and the black dots indicating the non-differentially expressed genes.

Functional enrichment analysis. The significantly enriched KEGG pathways of down- and upregulated genes were separately analyzed. As a result, a total of $25 \mathrm{KEGG}$ pathways that significantly associated with substance metabolism, such as insulin signaling pathway, 2-Oxocarboxylic acid metabolism were significantly enriched in downregulated genes (Fig. 2A). However, upregulated genes were demonstrated to be involved in 17 KEGG pathways associated with cancer development and cell activity, including the p53 signaling pathway, and cell cycle (Fig. 2B).

GO terms enrichment analysis for DEGs was performed and 173 significantly enriched GO terms were identified. 
Table I. Microarray experiments used for meta-analysis.

Characteristic

\begin{tabular}{lcccr} 
GEO ID & Sample size used/total & Case & Control & Platform \\
\cline { 3 - 4 } GSE34620 & $117 / 117$ & Ewing sarcoma (117) & NA & GPL570 \\
GSE17618 & $44 / 55$ & Ewing sarcoma (44) & NA & GPL570 \\
& (exclude cell line) & & & 220847514 \\
GSE17674 & $62 / 62$ & Ewing sarcoma (44) & Skeletal muscle (18) & GPL570 \\
\hline
\end{tabular}

ES, Ewing sarcoma; GEO, gene expression omnibus.
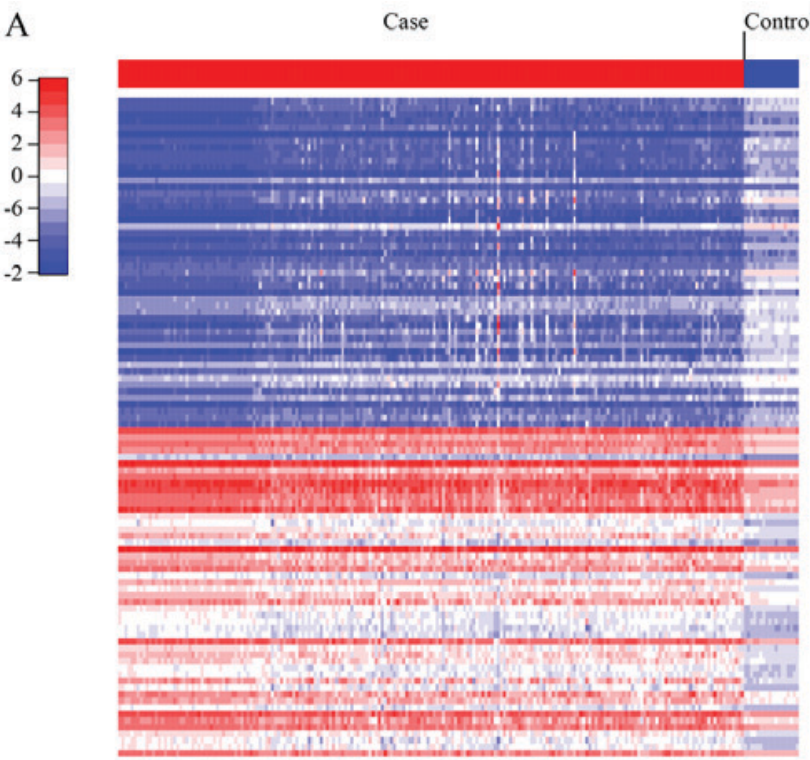

B

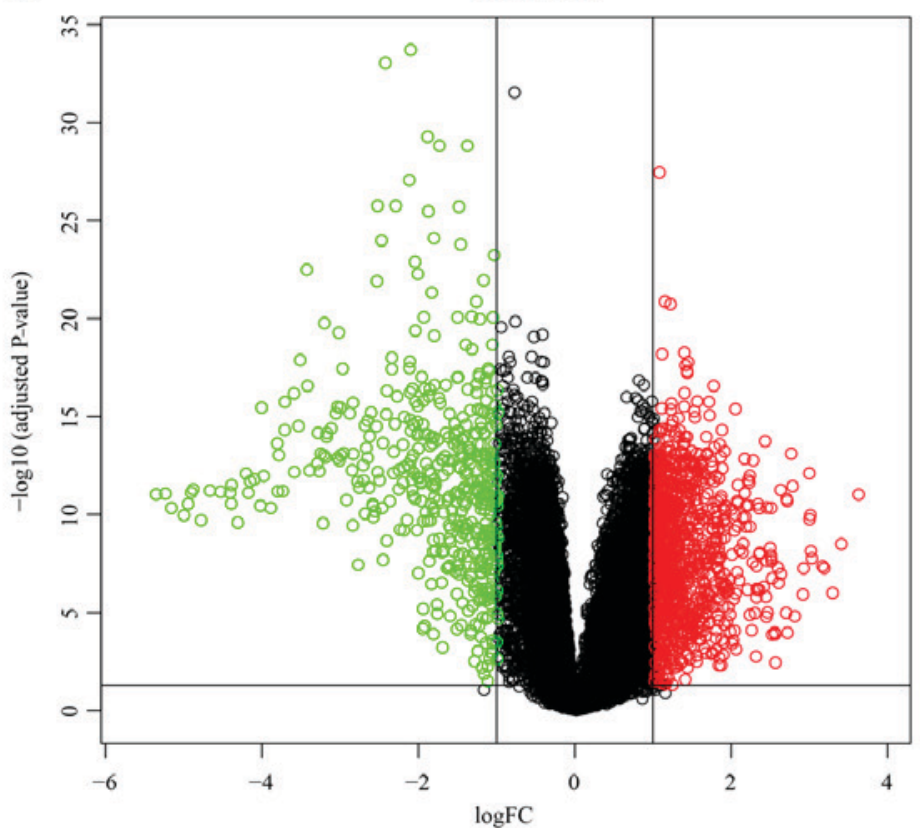

Figure 1. Differential expression analysis. (A) Heatmap of expression levels of the top 100 most significant DEGs. Horizontal and vertical axis represent genes and samples respectively and blue and red color represent low and high expression levels, respectively. Color bar at the top of the heatmap indicates sample type with red and blue indicating the ES and normal samples. (B) Volcano plot of the genes. X and Y axis is $\log 2$ Fold Change and -log10-based adjusted P-value. Green and red dots are the down-regulated and up-regulated genes, respectively and black dots are the non-differential expressed genes. DEG, differentially expressed genes; ES, Ewing sarcoma.

For BP terms, their association through the enrichment Map plug-in of Cytoscape software was explored. As a result, five clusters were obtained, which were associated with muscle contraction, cell division, extracellular matrix organization, response to stimuli and metabolism, respectively (Fig. 2C).

Network analysis. Combined analysis of network of PINA and a study by Menche et al (23) identified 13,259 interaction pairs among the 1,470 DEGs. Modular analysis obtained a total of six network modules contained five upregulated and one downregulated module as shown in Fig. 3. For interpretation of the biological processes of every module, KEGG pathway analysis for module genes through KOBAS online tool (kobas.cbi.pku.edu.cn/) was conducted (25). As a result, besides cancer-associated pathways, KEGG pathways associated with nervous system diseases, such as Parkinson's disease, Huntington's disease, were also significantly enriched in several network modules (Table II).

Kaplan-Meier analysis. To identify potential biomarkers involved in ES progression, simultaneously with network degree $>10$ (genes that directly interact with $\geq 10$ other genes in the network) and $\mid \log 2$ Fold Changel $>2$ were screened. As a result, 22 genes were obtained (Table III) which contained 18 down- and 4 upregulated genes Fig. 4 demonstrates their expression profiles in normal and ES samples. Kaplan-Meier analysis of the 22 genes based on the ES dataset from TCGA identified five genes, including PYGM, MEF2C, TRIM63, $B U B 1 B$ and $R A C G A P 1$, which are significantly associated with ES OS (Fig. 5; P<0.05) in the present study. Consistent with the differential expression analysis, upregulation of the three downregulated genes, PYGM, MEF2C, TRIM63, is associated with good ES prognosis, while, upregulation of the two 
A

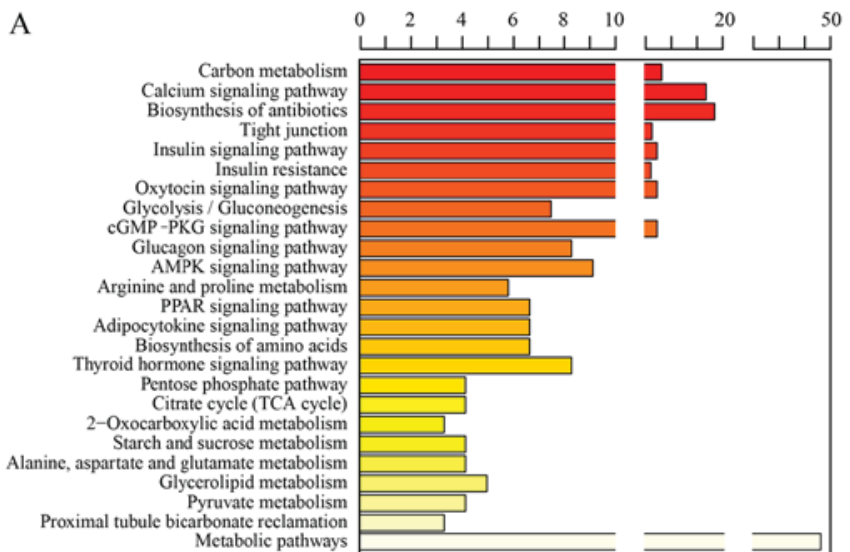

B

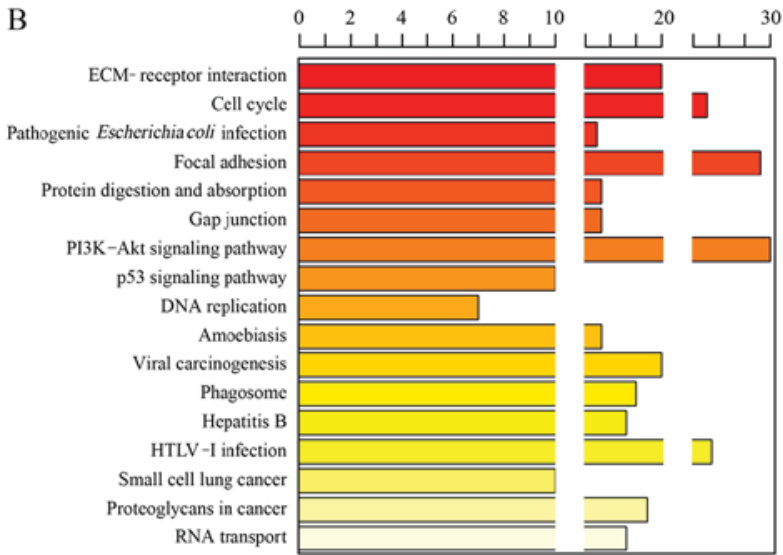

$\mathrm{C}$

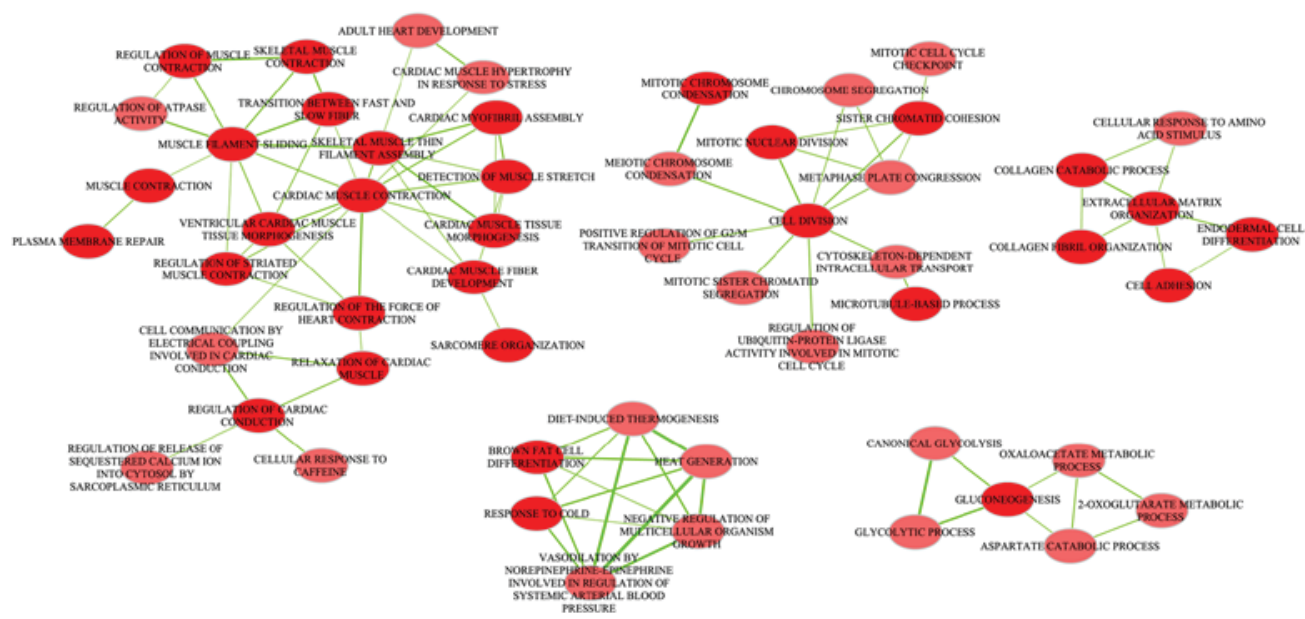

Figure 2. Functional enrichment analysis. (A) Significantly enriched signaling pathways of downregulated genes and (B) upregulated genes. Vertical axis is the gene number contained in the corresponding signaling pathway. The bar color indicates significance with the darker representing the more significant ones. (C) Crosstalk analysis of the BP terms of DEGs. Node and edge represent terms and interactions between two terms. Darker node color indicates more significance and larger node represents more genes contained in each term. Thicker edge indicates more overlapping genes between two terms. BP, biological processes; DEG, differentially expressed genes.

upregulated genes, i.e., $B U B 1 B$ and $R A C G A P 1$, are associated with poor ES prognosis (Fig. 5), which should provide valuable diagnosis and treatment biomarkers for ES.

$R T$-qPCR. Expression differences of $P Y G M, M E F 2 C$, TRIM63, BUB1B and RACGAP1 between the ES cell lines A673 and normal cell lines MSC were investigated using RT-qPCR for their significant associations with OSCC's OS. Consistent with results from the microarray analysis, $P Y G M$, $M E F 2 C$ and TRIM63 were downregulated, and BUBIB and $R A C G A P 1$ were upregulated in the ES cell line compared with the normal cells (Fig. 6).

\section{Discussion}

Ewing sarcoma is the second most frequent bone malignancy in children and adolescents (26). Chemotherapy and surgery are currently the main therapeutic modalities for ES (27). Despite aggressive therapy, the OS of ES patients is still dismal. The cure rate could be notably improved by the identification of molecular markers to aid the effective early diagnosis of malignancy and the prevention of tumor metastasis. In the present study, a meta-analysis of the transcriptomes of ES samples from three gene expression microarray datasets was performed, and 1,470 DEGs were identified, consisting of 984 up- and 486 downregulated genes. Using the DEGs, an ES disease network was constructed and six ES-associated disease clusters were obtained. Survival analysis identified five genes that were significantly associated with the survival rate of patients with ES.

GO term enrichment and clustering analysis were conducted for the 1,470 DEGs, and the GO terms were divided into five groups according to their biological roles in cell metabolism. The five groups are involved in the following physiological processes: Muscle contraction and morphogenesis, cell mitotic nuclear division and microtubule-based process, cell adhesion, heat generation process, and gluconeogenesis. Advanced malignancies that are often associated with bone metastasis can cause skeletal muscle weakness; the skeletal muscle quality is associated with muscle contraction and morphogenesis (28). A previous study demonstrated that metastasis-induced transforming growth factor (TGF)- $\beta$ release from bone contributes to muscle weakness by decreasing $\mathrm{Ca}^{2+}$-induced muscle force production (29). Cell mitosis is closely associated with tumor progression and metastasis; there, microtubules have been a major target for anticancer drugs development (30). 
Table II. Significantly enriched KEGG pathways of the six network modules.

A, Module 1

\begin{tabular}{lccc}
\hline Pathway & Pathway ID & P-value & FDR \\
\hline Parkinson's disease & hsa05012 & $6.01 \times 10^{-5}$ & $8.51 \times 10^{-4}$ \\
cGMP-PKG signaling pathway & hsa04022 & $9.64 \times 10^{-5}$ & $8.51 \times 10^{-4}$ \\
RNA transport & hsa03013 & $1.05 \times 10^{-4}$ & $8.51 \times 10^{-4}$ \\
Calcium signaling pathway & hsa04020 & $1.20 \times 10^{-4}$ & $8.51 \times 10^{-4}$ \\
Huntington's disease & hsa05016 & $1.47 \times 10^{-4}$ & $8.51 \times 10^{-4}$ \\
HTLV-I infection & hsa05166 & $3.44 \times 10^{-4}$ & $1.66 \times 10^{-3}$ \\
Ribosome biogenesis in eukaryotes & hsa03008 & $1.06 \times 10^{-3}$ & $4.38 \times 10^{-3}$ \\
Ribosome & hsa03010 & $2.47 \times 10^{-3}$ & $8.96 \times 10^{-3}$ \\
Viral carcinogenesis & hsa05203 & $5.30 \times 10^{-3}$ & $1.71 \times 10^{-2}$
\end{tabular}

B, Module 2

Spliceosome

African trypanosomiasis

Malaria

Pathogenic Escherichia coli infection

RNA degradation

Gap junction

mRNA surveillance pathway

NF-kappa B signaling pathway

AGE-RAGE signaling pathway in diabetic complications

TNF signaling pathway

Leukocyte transendothelial migration hsa03040

hsa05143

hsa05144

hsa05130

hsa03018

hsa04540

hsa03015

hsa04064

hsa04933

hsa04668

hsa04670
$3.82 \times 10^{-19}$

$1.35 \times 10^{-2}$

$1.87 \times 10^{-2}$

$2.09 \times 10^{-2}$

$2.90 \times 10^{-2}$

$3.30 \times 10^{-2}$

$3.45 \times 10^{-2}$

$3.49 \times 10^{-2}$

$3.78 \times 10^{-2}$

$4.11 \times 10^{-2}$

$4.40 \times 10^{-2}$
$6.87 \times 10^{-18}$

$6.87 \times 10^{-2}$

$6.87 \times 10^{-2}$

$6.87 \times 10^{-2}$

$6.87 \times 10^{-2}$

$6.87 \times 10^{-2}$

$6.87 \times 10^{-2}$

$6.87 \times 10^{-2}$

$6.87 \times 10^{-2}$

$6.87 \times 10^{-2}$

$6.87 \times 10^{-2}$

C, Module 3

RNA degradation

Parkinson's disease

Wnt signaling pathway

cGMP-PKG signaling pathway

Influenza A

Calcium signaling pathway

Huntington's disease

HTLV-I infection hsa03018

hsa05012

hsa04310

hsa04022

hsa05164

hsa04020

hsa05016

hsa05166
$9.77 \times 10^{-3}$

$1.79 \times 10^{-2}$

$1.80 \times 10^{-2}$

$2.09 \times 10^{-2}$

$2.21 \times 10^{-2}$

$2.26 \times 10^{-2}$

$2.42 \times 10^{-2}$

$3.23 \times 10^{-2}$
$2.76 \times 10^{-2}$

$2.76 \times 10^{-2}$

$2.76 \times 10^{-2}$

$2.76 \times 10^{-2}$

$2.76 \times 10^{-2}$

$2.76 \times 10^{-2}$

$2.76 \times 10^{-2}$

$3.23 \times 10^{-2}$

D, Module 4

\section{Cell cycle}

DNA replication

Pathogenic Escherichia coli infection

Gap junction

Phagosome

MicroRNAs in cancer hsa04110

hsa03030

hsa05130

hsa04540

hsa04145

hsa05206
$7.15 \times 10^{-8}$

$2.48 \times 10^{-7}$

$8.33 \times 10^{-7}$

$3.89 \times 10^{-4}$

$1.17 \times 10^{-3}$

$4.21 \times 10^{-3}$
$2.29 \times 10^{-6}$

$3.96 \times 10^{-6}$

$8.88 \times 10^{-6}$

$3.11 \times 10^{-3}$

$7.51 \times 10^{-3}$

$2.25 \times 10^{-2}$

\section{E, Module 5}

Cysteine and methionine metabolism

Pathogenic Escherichia coli infection

Gap junction

Apoptosis

Phagosome hsa00270

hsa05130

hsa04540

hsa04210

hsa04145
$6.92 \times 10^{-3}$

$8.42 \times 10^{-3}$

$1.34 \times 10^{-2}$

$2.11 \times 10^{-2}$

$2.33 \times 10^{-2}$
$2.95 \times 10^{-2}$

$2.95 \times 10^{-2}$

$3.12 \times 10^{-2}$

$3.26 \times 10^{-2}$

$3.26 \times 10^{-2}$ 
Table II. Continued.

\section{F, Module 6}

\begin{tabular}{llll}
\hline Pathway & Pathway ID & P-value & FDR \\
\hline Oocyte meiosis & hsa04114 & $3.67 \times 10^{-6}$ & $2.25 \times 10^{-5}$ \\
Cell cycle & hsa04110 & $3.75 \times 10^{-6}$ & $2.25 \times 10^{-5}$ \\
HTLV-I infection & hsa05166 & $3.28 \times 10^{-5}$ & $1.31 \times 10^{-4}$ \\
Ubiquitin mediated proteolysis & hsa04120 & $5.36 \times 10^{-4}$ & $9.19 \times 10^{-4}$ \\
Thyroid cancer & hsa05216 & $7.52 \times 10^{-3}$ & $1.00 \times 10^{-2}$ \\
Progesterone-mediated oocyte maturation & hsa04914 & $2.46 \times 10^{-2}$ & $2.95 \times 10^{-2}$ \\
Calcium signaling pathway & hsa04020 & $4.46 \times 10^{-2}$ & $4.86 \times 10^{-2}$ \\
Pathways in cancer & hsa05200 & $9.57 \times 10^{-2}$ & $9.57 \times 10^{-2}$ \\
\hline
\end{tabular}

FDR, false discovery data; KEGG, Kyoto Encyclopedia of Genes and Genome.

Cluster 1

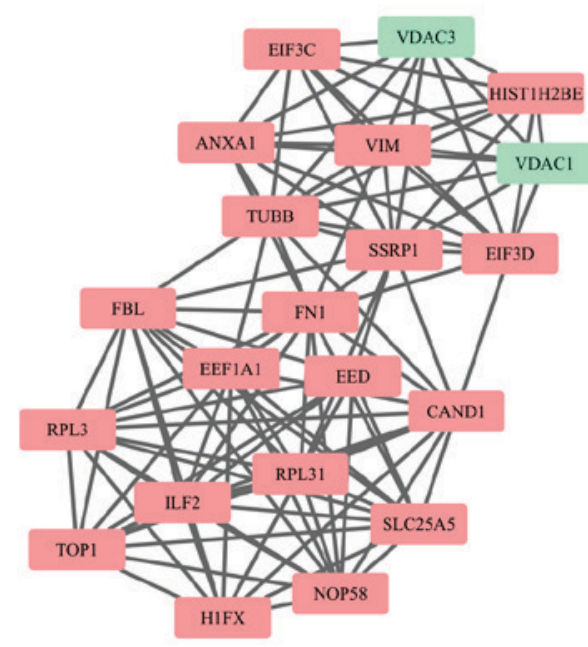

Cluster 4

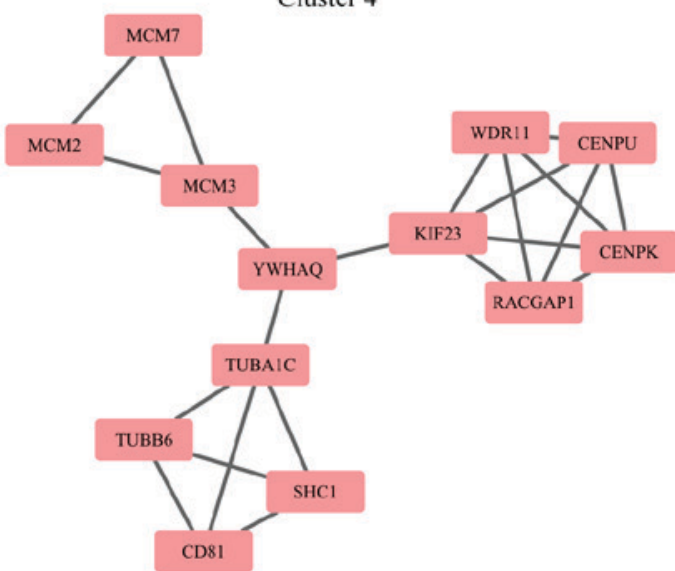

Cluster 2

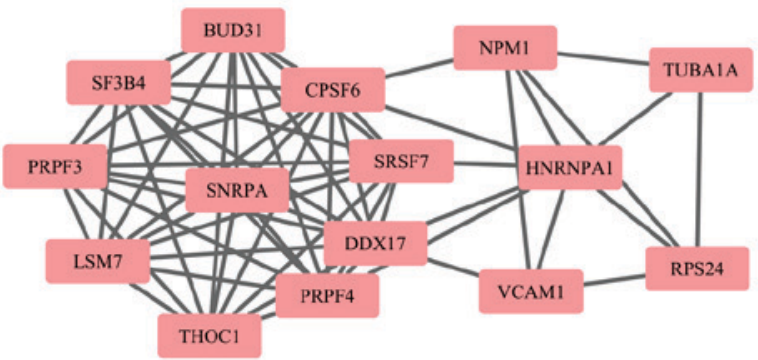

Cluster 3

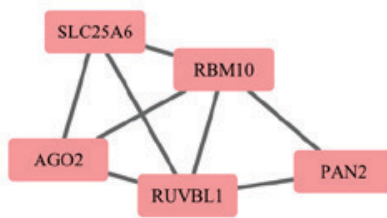

Cluster 5

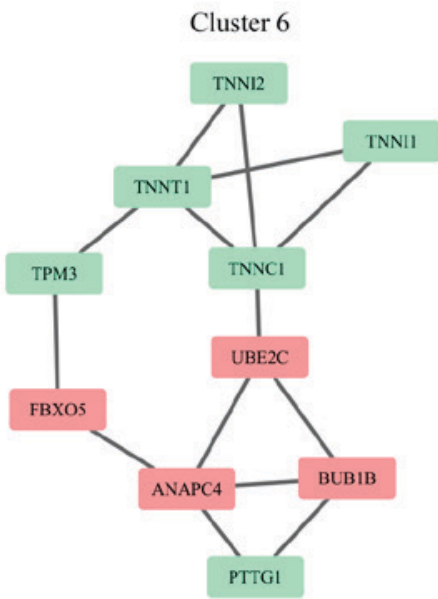

Figure 3. Modular analysis of network. Node and edge represent genes and interactions among genes. Green and red color indicate down-regulation and up-regulation respectively.

Physiological thermogenesis is beneficial for damaging tumor tissues and improving the clinical outcomes of patients with cancer, while hyperthermia has a direct killing effect on tumor cells and can have an inhibitory effect on tumor metastasis (31). Gluconeogenesis is a metabolic process whereby the body generates glucose from non-carbohydrate carbon source 
Table III. A total of 22 genes with degree $>10$ and $\mid \log 2$ (Fold-change) $\mid>2$.

\begin{tabular}{|c|c|c|c|}
\hline Gene symbol & Full name & $\log \mathrm{FC}$ & Degree \\
\hline ACTA1 & Actin, $\alpha 1$, skeletal muscle & -5.18 & 31 \\
\hline TNNT1 & Troponin T1, slow skeletal type & -4.19 & 15 \\
\hline PYGM & Glycogen phosphorylase, muscle associated & -4.02 & 15 \\
\hline MYBPC2 & Myosin binding protein $\mathrm{C}$, fast type & -3.91 & 13 \\
\hline NEB & Nebulin & -3.31 & 14 \\
\hline FHL1 & Four and A half LIM domains 1 & -3.23 & 11 \\
\hline TCAP & Titin-Cap & -3.13 & 11 \\
\hline TRIM63 & Tripartite motif containing 63 & -3.01 & 27 \\
\hline RYR1 & Ryanodine receptor 1 & -2.94 & 10 \\
\hline TTN & Titin & -2.90 & 36 \\
\hline ACTN2 & Actinin $\alpha 2$ & -2.86 & 16 \\
\hline SGCG & Sarcoglycan $\gamma$ & -2.68 & 14 \\
\hline CRYAB & Crystallin $\alpha$ B & -2.59 & 14 \\
\hline EEF1A2 & Eukaryotic translation elongation factor $1 \alpha 2$ & -2.57 & 11 \\
\hline ENO3 & Enolase 3 & -2.13 & 11 \\
\hline $\mathrm{MEF} 2 \mathrm{C}$ & Myocyte enhancer factor $2 \mathrm{C}$ & -2.06 & 10 \\
\hline PRKCQ & Protein kinase $\mathrm{C} \theta$ & -2.06 & 19 \\
\hline FLNC & Filamin C & -2.02 & 16 \\
\hline RACGAP1 & Ras GTPase activating protein 1 & 2.15 & 10 \\
\hline BUB1B & BUB1 mitotic checkpoint serine/threonine kinase B & 2.20 & 12 \\
\hline $\mathrm{EZH} 2$ & Enhancer of zeste 2 polycomb repressive complex 2 subunit & 2.61 & 31 \\
\hline CCND1 & Cyclin D1 & 2.75 & 16 \\
\hline
\end{tabular}

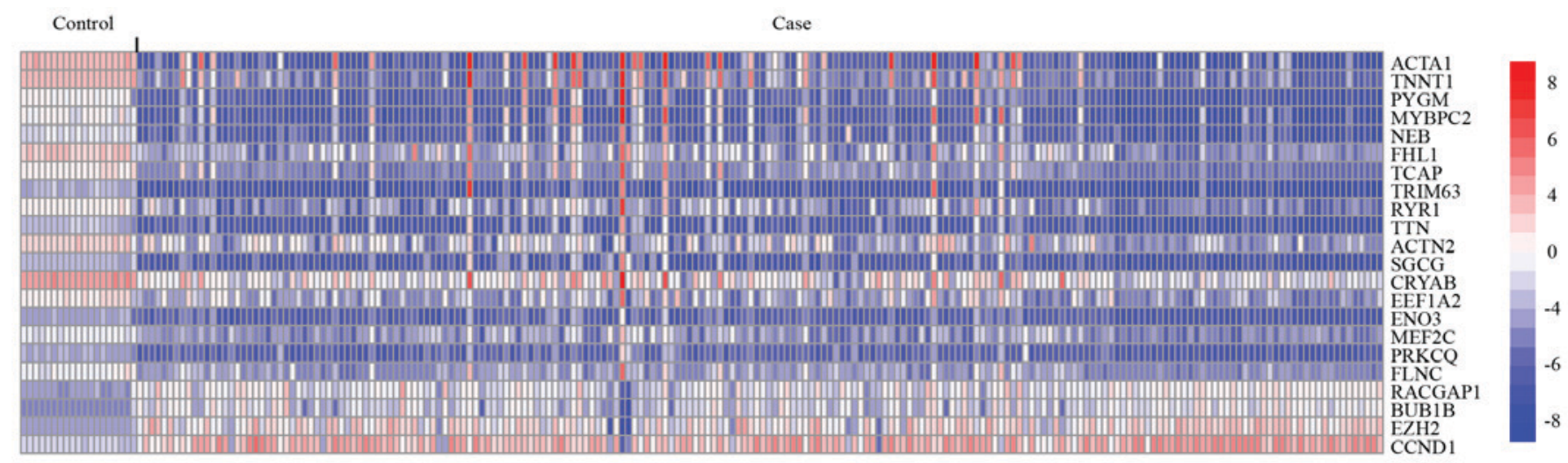

Figure 4. Heatmap of the 22 genes with network degree $>10$ and llog2Fold-Changel $>2$. Horizontal and vertical axis represent genes and samples, respectively and green and red color indicate low and high expression levels, respectively.

and provides energy for the growth and survival of cells (32). Studies have suggested that gluconeogenesis could cause a metabolic stress and therefore disrupt the metabolic rewiring of cancer cells $(33,34)$. The five GO term groups identified in the present study, are all involved in cancer progression and metastasis, representing the typical ES progression-associated biological processes $(35,36)$.

As previously stated, based on the 1,470 DEGs, an ES disease network was constructed, and six disease clusters were ultimately identified. Several cancer-associated signaling pathways and metabolic processes were observed by enrichment analysis for the DEGs in each cluster. The cGMP-PKG signaling pathway was significantly enriched in the DEGs of clusters 1 and 3 and proved to be closely associated with tumor progression. Upregulated cGMP and its downstream protein kinase $\mathrm{G}(\mathrm{PKG})$ are known to inhibit the proliferation and induce the apoptosis of colon cancer cells, and activated intracellular cGMP-PKG pathway is known to enhance the degradation of $\beta$-catenin in SW480 colon cancer cells (37-39). Calcium signaling pathways were observed in clusters 1,3 and 6; these signaling pathways serve a significant role in the cell apoptosis process (35). Under pathological conditions, the $\mathrm{Ca}^{2+}$ level is markedly increased in many types of cells, resulting in the enhanced expression of pro-apoptotic factors (40). 

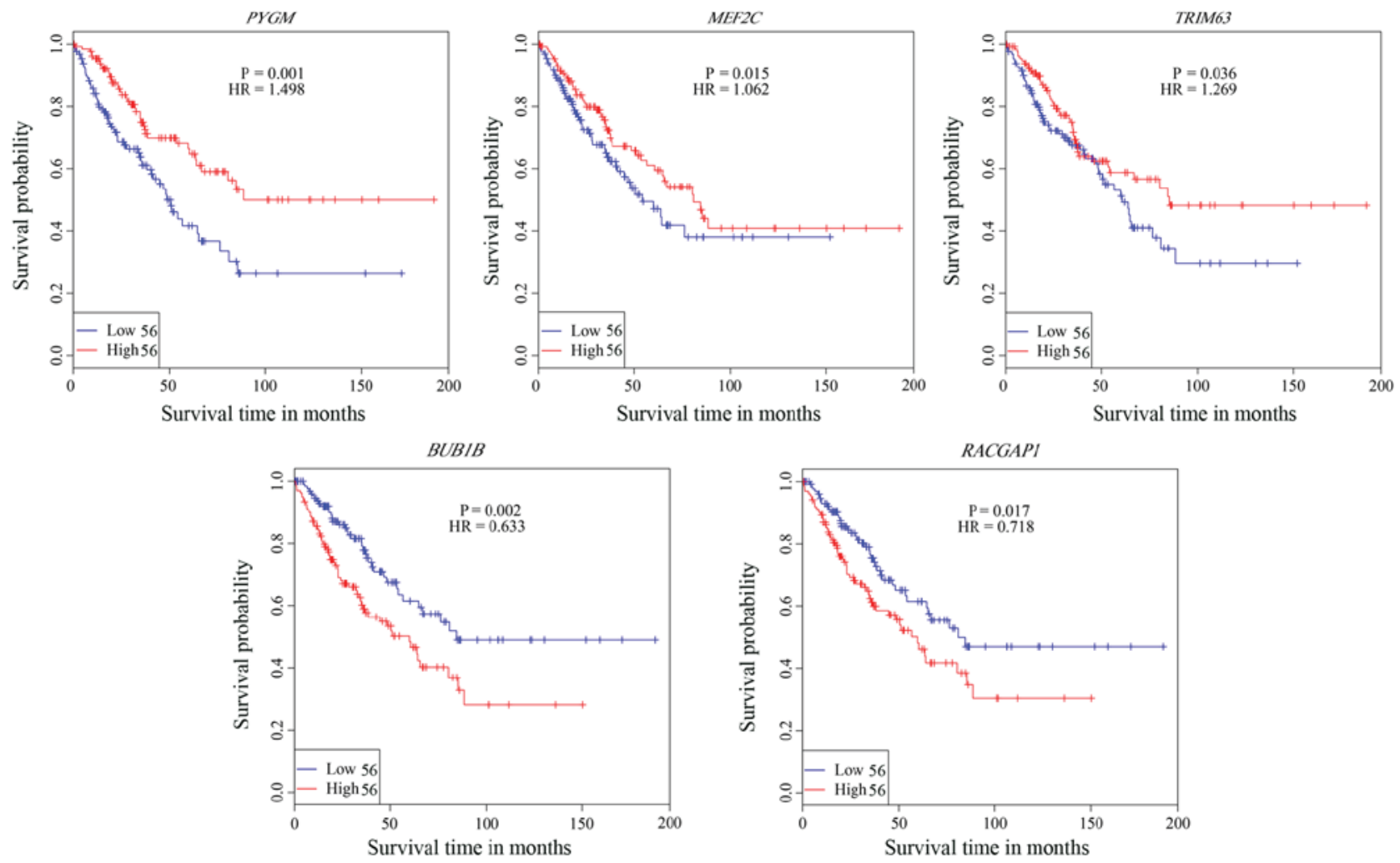

Figure 5. Overall survival curves of the five genes significantly associated with ES prognosis obtained through the Kaplan-Meier analysis. Blue and red curve are samples with lower and higher expression levels than the median, respectively. Plus signs are censored values. The P-value was obtained through log-rank test. ES, Ewing sarcoma; HR represents hazard ratio.

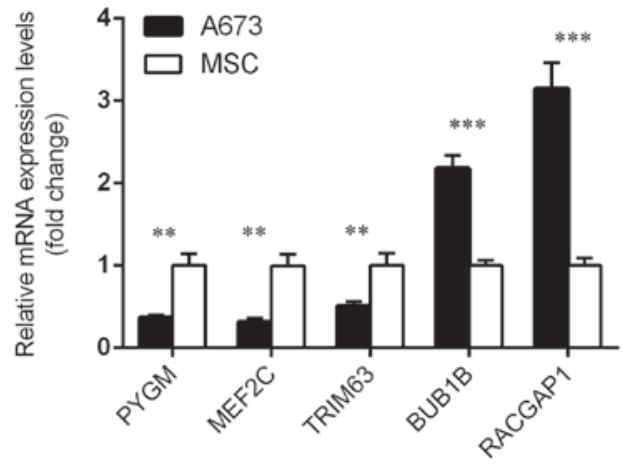

Figure 6. Validation of the data with RT-qPCR. The five genes identified in microarray data were validated by RT-qPCR. BUB1B, budding uninhibited by benzimidazoses1; MEF2C, myocyte-specific enhancer factor $2 \mathrm{C}$; PYGM, glycogen phosphorylase; RACGAP, ras GTPase-activating protein 1 , TRIM63, Tripartite Motif Containing $63 .{ }^{* *} \mathrm{P}<0.01$ and ${ }^{* * *} \mathrm{P}<0.001$ A673 vs. MSC group.

Curcumin, a traditional Chinese medicine, may induce cell apoptosis through upregulating the $\mathrm{Ca}^{2+}$ level in lung cancer cells $(35,41,42)$. Certain disease-type markers, including Parkinson's disease (PD) associated genes [VDAC1 (43), VDAC3 (44)], were observed in clusters 1 and 3. Many studies have demonstrated that the cancer incidence in patients with $\mathrm{PD}$ is significantly lower than in the patients without PD, but the detailed mechanism remains to be explored (45). Many DEGs involved in the cell cycle process were in clusters 4 and 6 . Cell proliferation is an essential mechanism for the growth, development and regeneration of eukaryotic organisms. Therefore, targeting the cell cycle process to regulate cell proliferation has been one of the most effective approaches to treat cancers (46). Other signaling pathways or metabolic processes such as the mRNA surveillance pathway, the AGE-RAGE signaling pathway in diabetic complications, RNA degradation, cysteine and methionine metabolism and others were also observed in certain clusters. These signaling pathways or metabolic processes observed by enrichment analysis of the DEGs may participate in the progression and metastasis of ES and could also be considered potential molecular targets for early diagnosis and therapy $(35,36)$.

Survival analysis revealed that five DEGs were significantly associated with the survival rates of ES patients. $B U B 1 B$ and $R A C G A P 1$ were markedly upregulated in the ES samples. $B U B 1 B$, a member of the spindle assembly checkpoint protein family, has been associated with many types of cancer $(47,48)$. Upregulated expression of $B U B 1 B$ enhanced the proliferation, migration, and invasion ability of prostate cancer cell lines (48). RACGAP1 is a component of the central spindle and essential for the induction of cytokinesis. Overexpressed $R A C G A P 1$ was associated with poor disease-free and overall survival, and may act as an independent predictive marker for lymph node metastasis, recurrence and poor prognosis of colorectal cancer (49-51). The present results demonstrated that the expression of $B U B 1 B$ and RACGAPI in ES samples is negatively associated with the survival rates of ES patients. In addition, the downregulated expression of PYGM, MEF $2 C$, and TRIM63 was also observed in the present study, with their expression being positively associated with the survival rates of ES patients. The present results suggest that $B U B 1 B$, 
RACGAP1, PYGM, MEF2C, and TRIM63 may be considered potential markers for the prognosis of ES, but this needs to be investigated further.

In summary, the transcriptomes of ES samples from three independent gene expression chips was investigated and six disease clusters based on the 1,470 DEGs was constructed. Several cancer-associated signaling pathways, metabolic processes, or disease types were identified by the enrichment analysis, that could act as potential markers for early diagnosis and as targets for therapy. Survival analysis revealed that five DEGs were significantly associated with the survival rates of ES patients and could be considered predictive markers for the prognosis of ES, but this needs to be investigated further.

In the present study, a comprehensive analysis of three ES-associated microarray datasets was conducted and several KEGG pathways and GO term clusters that may be involved in ES progression were obtained. Additionally, five genes that are significantly associated with OS of patients with ES were identified, which may be helpful for ES early diagnosis and treatment, but this needs to be validated in future studies.

\section{Acknowledgements}

Not applicable.

\section{Funding}

No funding was received.

\section{Availability of data and materials}

The datasets generated and/oranalyzed during the current study are available in the NCBI repository: https://www.ncbi.nlm. nih.gov/geo/query/acc.cgi?acc=GSE34620; https://www.ncbi. nlm.nih.gov/geo/query/acc.cgi?acc=GSE17618; https:/www. ncbi.nlm.nih.gov/geo/query/acc.cgi?acc=GSE17674.

\section{Authors' contributions}

YXQ put forward the ideas of this article, wrote this article and analyzed the data. SJB helped revise the manuscript, analyzed the data and put forward ideas for the article. ZHY helped with acquisition of data, and analysis and interpretation of data. WS provided valuable instructions and the figure combinations, analyzed the data and study design, and helped revising the manuscript. All authors read and approved the final manuscript.

\section{Ethics approval and consent to participate}

Not applicable.

\section{Patient consent for publication}

Not applicable.

\section{Competing interests}

The authors declare that they have no competing interests.

\section{References}

1. Balamuth NJ and Womer RB: Ewing's sarcoma. Lancet Oncol 11: 184-192, 2010.

2. Sand LG, Szuhai K and Hogendoorn PC: Sequencing overview of ewing sarcoma: A journey across genomic, epigenomic and transcriptomic landscapes. Int J Mol Sci 16: 16176-16215, 2015.

3. Kovar H, Alonso J, Aman P, Aryee DN, Ban J, Burchill SA, Burdach S, De Alava E, Delattre O, Dirksen U, et al: The first European interdisciplinary ewing sarcoma research summit. Front Oncol 2: 54, 2012.

4. Paulussen M, Ahrens S, Burdach S, Craft A Dockhorn-Dworniczak B, Dunst J, Fröhlich B, Winkelmann W, Zoubek A and Jürgens H: Primary metastatic (stage IV) Ewing tumor: Survival analysis of 171 patients from the EICESS studies. European intergroup cooperative ewing sarcoma studies. Ann Oncol 9: 275-281, 1998.

5. Postel-Vinay S, Veron AS, Tirode F, Pierron G, Reynaud S, Kovar H, Oberlin O, Lapouble E, Ballet S, Lucchesi C, et al: Common variants near TARDBP and EGR2 are associated with susceptibility to Ewing sarcoma. Nat Genet 44: 323-327, 2012.

6. Delattre O,Zucman J, Plougastel B, Desmaze C, Melot T, Peter M, Kovar H, Joubert I, de Jong P, Rouleau G, et al: Gene fusion with an ETS DNA-binding domain caused by chromosome translocation in human tumours. Nature 359: 162-165, 1992.

7. Delattre O,Zucman J, Melot T, Garau XS, Zucker JM, Lenoir GM, Ambros PF, Sheer D, Turc-Carel C, Triche TJ, et al: The Ewing family of tumors-a subgroup of small-round-cell tumors defined by specific chimeric transcripts. N Engl J Med 331: 294-299, 1994.

8. Sorensen PH, Lessnick SL, Lopez-Terrada D, Liu XF, Triche TJ and Denny CT: A second Ewing's sarcoma translocation, $\mathrm{t}(21 ; 22)$, fuses the EWS gene to another ETS-family transcription factor, ERG. Nat Genet 6: 146-151, 1994.

9. Potratz J, Dirksen U, Jurgens H and Craft A: Ewing sarcoma: Clinical state-of-the-art. Pediatr Hematol Oncol 29: 1-11, 2012.

10. Jeon IS, Davis JN, Braun BS, Sublett JE, Roussel MF, Denny CT and Shapiro DN: A variant Ewing's sarcoma translocation $(7 ; 22)$ fuses the EWS gene to the ETS gene ETV1. Oncogene 10: 1229-1234, 1995.

11. Kaneko Y, Yoshida K, Handa M, Toyoda Y, Nishihira H, Tanaka Y, Sasaki Y, Ishida S, Higashino F and Fujinaga K: Fusion of an ETS-family gene, EIAF, to EWS by $\mathrm{t}(17 ; 22)(\mathrm{q} 12 ; \mathrm{q} 12)$ chromosome translocation in an undifferentiated sarcoma of infancy. Genes Chromosomes Cancer 15: 115-121, 1996.

12. Peter M, Couturier J, Pacquement H, Michon J, Thomas G, Magdelenat $\mathrm{H}$ and Delattre O: A new member of the ETS family fused to EWS in Ewing tumors. Oncogene 14: 1159-1164, 1997.

13. Folpe AL, Hill CE, Parham DM, O'Shea PA and Weiss SW: Immunohistochemical detection of FLI-1 protein expression: A study of 132 round cell tumors with emphasis on CD99-positive mimics of Ewing's sarcoma/primitive neuroectodermal tumor. Am J Surg Pathol 24: 1657-1662, 2000.

14. Rossi S, Orvieto E, Furlanetto A, Laurino L, Ninfo V and Dei Tos AP: Utility of the immunohistochemical detection of FLI-1 expression in round cell and vascular neoplasm using a monoclonal antibody. Mod Pathol 17: 547-552, 2004.

15. Lin O, Filippa DA and Teruya-Feldstein J: Immunohistochemical evaluation of FLI-1 in acute lymphoblastic lymphoma (ALL): A potential diagnostic pitfall. Appl Immunohistochem Mol Morphol 17: 409-412, 2009.

16. Savola S, Klami A, Myllykangas S, Manara C, Scotlandi K, Picci P, Knuutila S and Vakkila J: High expression of complement component 5 (C5) at tumor site associates with superior survival in Ewing's sarcoma family of tumour patients. ISRN Oncol 2011: 168712, 2011.

17. Leek JT, Johnson WE, Parker HS, Jaffe AE and Storey JD: The sva package for removing batch effects and other unwanted variation in high-throughput experiments. Bioinformatics 28: 882-883, 2012.

18. Diboun I, Wernisch L, Orengo CA and Koltzenburg M: Microarray analysis after RNA amplification can detect pronounced differences in gene expression using limma. BMC Genomics 7: 252, 2006.

19. Huang da W, Sherman BT and Lempicki RA: Systematic and integrative analysis of large gene lists using DAVID bioinformatics resources. Nat Protoc 4: 44-57, 2009.

20. Merico D, Isserlin R, Stueker O, Emili A and Bader GD: Enrichment map: A network-based method for gene-set enrichment visualization and interpretation. PLoS One 5: e13984, 2010. 
21. Kohl M, Wiese S and Warscheid B: Cytoscape: Software for visualization and analysis of biological networks. Methods Mol Biol 696: 291-303, 2011

22. Cowley MJ, Pinese M, Kassahn KS, Waddell N, Pearson JV, Grimmond SM, Biankin AV, Hautaniemi S and Wu J: PINA v2.0: Mining interactome modules. Nucleic Acids Res 40 (Database Issue): D862-D865, 2012.

23. Menche J, Sharma A, Kitsak M, Ghiassian SD, Vidal M, Loscalzo J and Barabási AL: Disease networks. Uncovering disease-disease relationships through the incomplete interactome. Science 347: 1257601, 2015.

24. Livak KJ and Schmittgen TD: Analysis of relative gene expression data using real-time quantitative PCR and the 2(-Delta Delta C(T)) method. Methods 25: 402-408, 2001.

25. Xie C, Mao X, Huang J, Ding Y, Wu J, Dong S, Kong L, Gao G Li CY and Wei L: KOBAS 2.0: A web server for annotation and identification of enriched pathways and diseases. Nucleic Acids Res 39: W316-W322, 2011.

26. Hamanoue $S$ and Makimoto A: Ewing sarcoma. Gan To Kagaku Ryoho 34: 175-180, 2007 (In Japanese).

27. Ray-Coquard I and Le Cesne A: A role for maintenance therapy in managing sarcoma. Cancer Treat Rev 38: 368-378, 2012.

28. Fearon KC, Glass DJ and Guttridge DC: Cancer cachexia: Mediators, signaling, and metabolic pathways. Cell Metab 16: 153-166, 2012.

29. Waning DL, Mohammad KS, Reiken S, Xie W, Andersson DC, John S, Chiechi A, Wright LE, Umanskaya A, Niewolna M, et al: Excess TGF- $\beta$ mediates muscle weakness associated with bone metastases in mice. Nat Med 21: 1262-1271, 2015.

30. He Y, Yan D, Zheng D, Hu Z, Li H and Li J: Cell division cycle 6 promotes mitotic slippage and contributes to drug resistance in paclitaxel-treated cancer cells. PLoS One 11: e0162633, 2016.

31. Yan B, Ouyang R, Huang C, Liu F, Neill D, Li C and Dewhirst M: Heat induces gene amplification in cancer cells. Biochem Biophys Res Commun 427: 473-477, 2012.

32. Rui L: Energy metabolism in the liver. Compr Physiol 4: 177-197, 2014.

33. Ma R, Zhang W, Tang K, Zhang H, Zhang Y, Li D, Li Y, Xu P, Luo S, Cai W, et al: Switch of glycolysis to gluconeogenesis by dexamethasone for treatment of hepatocarcinoma. Nat Commun 4: 2508, 2013.

34. Khan MW and Chakrabarti P: Gluconeogenesis combats cancer: Opening new doors in cancer biology. Cell Death Dis 6: e1872, 2015.

35. Xu X, Chen D, Ye B, Zhong F and Chen G: Curcumin induces the apoptosis of non-small cell lung cancer cells through a calcium signaling pathway. Int J Mol Med 35: 1610-1616, 2015.

36. Ventura S, Aryee DN, Felicetti F, De Feo A, Mancarella C, Manara MC, Picci P, Colombo MP, Kovar H, Carè A and Scotlandi K: CD99 regulates neural differentiation of Ewing sarcoma cells through miR-34a-Notch-mediated control of NF-kB signaling. Oncogene 35: 3944-3954, 2016.

37. Tetsu $\mathrm{O}$ and McCormick F: Beta-catenin regulates expression of cyclin D1 in colon carcinoma cells. Nature 398: 422-426, 1999

38. Li H, Liu L, David ML, Whitehead CM, Chen M, Fetter JR, Sperl GJ, Pamukcu R and Thompson WJ: Pro-apoptotic actions of exisulind and CP461 in SW480 colon tumor cells involve beta-catenin and cyclin D1 down-regulation. Biochem Pharmacol 64: 1325-1336, 2002.
39. Ren Y, Zheng J, Yao X, Weng G and Wu L: Essential role of the $\mathrm{cGMP} / \mathrm{PKG}$ signaling pathway in regulating the proliferation and survival of human renal carcinoma cells. Int J Mol Med 34: 1430-1438, 2014

40. Lv Y, Fang M, Zheng J, Yang B, Li H, Xiuzigao Z, Song W, Chen Y and Cao W: Low-intensity ultrasound combined with 5-aminolevulinic acid administration in the treatment of human tongue squamous carcinoma. Cell Physiol Biochem 30: 321-333, 2012.

41. Seo SR and Seo JT: Calcium overload is essential for the acceleration of staurosporine-induced cell death following neuronal differentiation in PC12 cells. Exp Mol Med 41: 269-276, 2009.

42. Ma TS: Sarcoplasmic reticulum calcium ATPase overexpression induces cellular calcium overload and cell death. Ann N Y Acad Sci 853: 325-328, 1998 .

43. Narendra D, Kane LA, Hauser DN, Fearnley IM and Youle RJ: p62/SQSTM1 is required for Parkin-induced mitochondrial clustering but not mitophagy; VDAC1 is dispensable for both. Autophagy 6: 1090-1106, 2010.

44. Shamir R, Klein C, Amar D, Vollstedt EJ, Bonin M, Usenovic M, Wong YC, Maver A, Poths S, Safer H, et al: Analysis of blood-based gene expression in idiopathic Parkinson disease. Neurology 89: 1676-1683, 2017.

45. Bajaj A, Driver JA and Schernhammer ES: Parkinson's disease and cancer risk: A systematic review and meta-analysis. Cancer Causes Control 21: 697-707, 2010.

46. Diaz-Moralli S, Tarrado-Castellarnau M, Miranda A and Cascante M: Targeting cell cycle regulation in cancer therapy. Pharmacol Ther 138: 255-271, 2013.

47. Ding Y, Hubert CG, Herman J, Corrin P, Toledo CM, Skutt-Kakaria K, Vazquez J, Basom R, Zhang B, Risler JK, et al: Cancer-Specific requirement for BUB1B/BUBR1 in human brain tumor isolates and genetically transformed cells. Cancer Discov 3: 198-211, 2013.

48. Fu X, Chen G, Cai ZD, Wang C, Liu ZZ, Lin ZY, Wu YD, Liang YX, Han ZD, Liu JC and Zhong WD: Overexpression of BUB1B contributes to progression of prostate cancer and predicts poor outcome in patients with prostate cancer. Onco Targets Ther 9: 2211-2220, 2016.

49. Zhao WM and Fang G: MgcRacGAP controls the assembly of the contractile ring and the initiation of cytokinesis. Proc Natl Acad Sci USA 102: 13158-13163, 2005.

50. Kitamura T, Kawashima T, Minoshima Y, Tonozuka Y, Hirose K and Nosaka T: Role of MgcRacGAP/Cyk4 as a regulator of the small GTPase Rho family in cytokinesis and cell differentiation. Cell Struct Funct 26: 645-651, 2001

51. Imaoka H, Toiyama Y, Saigusa S, Kawamura M, Kawamoto A, Okugawa Y, Hiro J, Tanaka K, Inoue Y, Mohri Y and Kusunoki M: RacGAP1 expression, increasing tumor malignant potential, as a predictive biomarker for lymph node metastasis and poor prognosis in colorectal cancer. Carcinogenesis 36 : 346-354, 2015 .

This work is licensed under a Creative Commons Attribution-NonCommercial-NoDerivatives 4.0 International (CC BY-NC-ND 4.0) License. 